\title{
Pulling versus guiding: a modification of retrograde guided intubation
}

We describe a modification of retrograde guided intubation. With the help of a gliding knot fixed around the side hole of the tracheal tube, we use the catheter to pull and guide the tracheal tube down the larynx and trachea. The technique offers several advantages: it is surprisingly fast, relatively atraumatic, easy to perform, and eliminates most causes of failure.

Retrograde guided intubation is a technique occasionally employed when attempts at direct laryngeal exposure would be hazardous to the patient. Although convenient, the technique has not gained wide clinical acceptance for it requires considerable skill and is not without complications. ${ }^{1}$ The following case report describes a major modification to this technique that we devised and used in the management of a patient scheduled for an open reduction and fusion of a cervical spine fracture.

\section{Case report}

A 28-year-old patient, who two weeks earlier had sustained a fracture-dislocation at C7-T1, was scheduled for an open reduction and spinal fusion from C4-T2. Whilst breathing spontaneously, the patient exhibited paradoxical movements of the chest wall and abdomen. A sensory level was present at T2. Abdominal cutaneous, cremasteric, and lower extremity reflexes were absent. The CT scan showed a burst fracture at C7-T1 with anterior displacement and disruption of all three columns at that level. The chest radiograph was normal. The ECG revealed a sinus bradycardia.

\section{Key words}

ANAESTHESIA: neurosurgical, cervical spine fracture; INTUBATION, TRACHEAL: technique, retrograde, cricotracheal.

From the Department of Anaesthesia, Montreal Neurological Institute, McGill University, Montreal.

Address correspondence to: Dr. M.N. Abou-Madi, Department of Anaesthesia, Montreal Neurological Hospital, 3801 University Street, Montreal, Quebec H3A 2B4.
The patient, premedicated with lorazepam $2 \mathrm{mg}$, given sublingually, was placed in Cone-Barton tongs with $10 \mathrm{~kg}$ of traction. To prepare the patient for retrograde guided intubation, he was given atropine, $0.6 \mathrm{mg}$ IV. A ten per cent solution of cocaine was used to anaesthetise the mucous membrane of the nose. The superior laryngeal nerves were blocked bilaterally below the apex of the greater cornu of the hyoid bone, using a two per cent solution of lidocaine injected both superficially and deep to the thyrohyoid membrane. The patient was asked to gargle for two minutes with a four per cent aqueous solution of lidocaine. The skin over the cricotracheal membrane was infiltrated with a one per cent solution of lidocaine and the membrane punctured with a 16-ga Tuohy needle. A 19-ga epidural catheter was passed through the needle, threaded cephalad, and retrieved from the mouth. A second catheter was passed through the nose and taken out from the mouth. The ends of the two catheters protruding from the mouth were tied together. The nasal end of the second catheter was pulled to draw the first catheter from the mouth out of the nose.

At this stage, the following modification replaced the commonly employed step of threading the tracheal tube over the catheter into the trachea: rather than guiding the tube over the catheter, traction on the catheter from outside the cricotracheal membrane enabled us to draw as well as guide the tracheal tube towards the trachea. A gliding knot was fixed around the side hole of the tracheal tube. To make the knot, the nasal end of the catheter was passed through the side hole of the tracheal tube (outsidein), retrieved from the beveled end hole, looped over the lower end of the tube, reinserted through the side hole (outside-in), and fed upward into the tube until it protruded from the latter's upper end (Figure 1). Once retrieved, the tube and catheter were firmly held together. Intubation was attempted nasally, using the cricotracheal end of the catheter to pull on the tube. The tube curved smoothly downwards through the nose, passed the oropharynx, slipped into the laryngeal inlet, and easily entered the trachea (Figure 2). The position of the tube was confirmed by auscultation. The catheter was removed by cutting it at the puncture site. Because of the gliding 


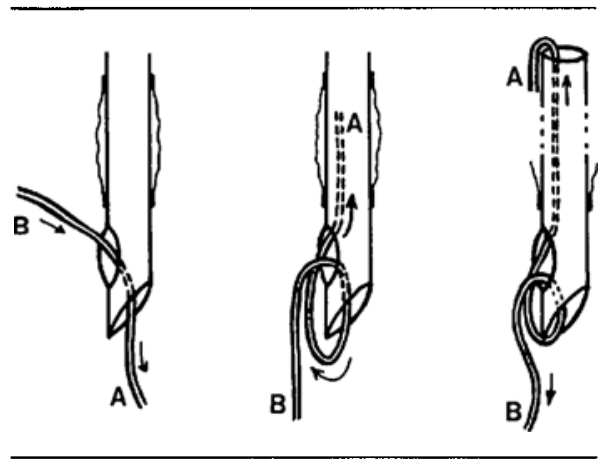

FIGURE I Lower end of tracheal tube and catheter. Arrows indicate course of catheter around the side hole.

nature of the knot, the catheter could be pulled out from the tracheal tube without difficulty. The tube was secured. Anaesthesia was maintained with pancuronium, nitrous oxide, isoflurane, and fentanyl. At the end of surgery, the trachea was extubated uneventfully.

\section{Discussion}

A major challenge in the anaesthetic management of patients with unstable cervical spine fractures is to maintain complete neck immobility during tracheal intubation. Because of their precarious cardiovascular and respiratory function, these patients cannot tolerate heavy preoperative sedation. Faced with such a restrictive clinical situation, anaesthetists have developed several techniques to intubate the trachea safely. These include blind nasal intubation, fibreoptic guided intubation, and retrograde guided intubation. Unfortunately, none of these has proved ideal despite the modifications that have been developed. ${ }^{2-11}$

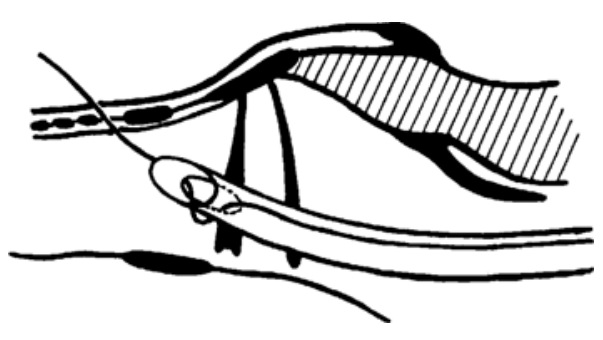

FIGURE 2 Cross-section of larynx and trachea, with the tracheal tube pulled down by the catheter guide into correct position. The catheter exit is at the level of the cricotracheal membrane.
Awake intubation is usually indicated for faciomaxillary deformities, poor mask-to-face fitting, facial injuries, decreased airway patency from inflammatory or neoplastic pathology, difficult laryngoscopy, limited neck movements, cervical instability from injuries and other causes, and for vomiting and aspiration.

Blind nasal intubation has been largely abandoned because of its low reported success rate and its tendency to produce airway damage, inducing epistaxis, blood aspiration, and respiratory obstruction. In addition, it sometimes precipitates breath holding and hypoxia.

As an alternative, the fibreoptic endoscope has recently gained popularity. Its limitations, however, are multiple and crucial. The operator requires both skill with fibreoptic equipment and experienced assistants. The fibreoptic endoscope is difficult to use in the presence of distorted airway anatomy, laryngeal pathology, decreased space between the edge of the epiglottis and the posterior pharyngeal wall, and bloody secretions. Progression of the fibreoptic scope may be impeded at the laryngeal inlet. A tracheal tube large enough to accommodate the endoscope must be used. The equipment is fragile and expensive.

Compared with fibreoptic endoscopy, retrograde guided intubation is easy to learn, does not require complex, expensive apparatus, can be used in emergency situations by personnel not proficient in endoscopy, and is particularly useful in the presence of oropharyngeal bleeding which may obscure the field of the fibreoptic laryngoscope and make its use extremely difficult. Moreover, complications such as epistaxis, bleeding at the site of puncture, sheared catheters, subcutaneous emphysema, and infection are either minor or preventable.

Unfortunately, difficulties were met in intubating the trachea despite the guiding catheter. The tube kinked, impinged on the epiglottis, was held at the larynx, or entered the oesophagus. To overcome the problem, the technique has been modified in several ways. Bourke and Levesque passed the guide through the side hole of the tracheal tube, ${ }^{6}$ Roberts used a Swan-Ganz introducer wire to replace the catheter guide, ${ }^{7}$ and Tobias advanced a fibreoptic laryngoscope into the trachea. ${ }^{9}$ Freund passed an Eschmann stylet over the epidural catheter to make it more rigid. ${ }^{11}$

Because of obvious anatomical landmarks and casy accessibility, anaesthetists have, to date, selected the cricothyroid membrane site to insert their catheter guides. ${ }^{3-11}$ We prefer the cricotracheal membrane puncture for the following reasons. First, it avoids the cavity of the larynx which extends from the laryngeal inlet to the level of the lower border of the cricoid cartilage. The anterior part of the cricovocal membrane, or cricothyroid ligament, is thick, strong, and subcutaneous. It connects the con- 


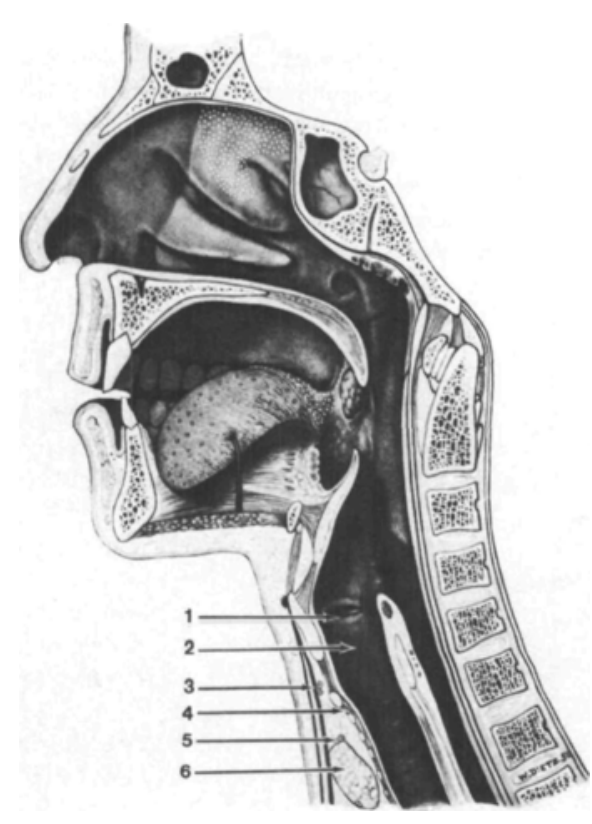

FIGURE 3 Midsagittal section through the neck and adjoining head and thoracic regions. 1-Vocal cord. 2-Laryngeal cavity. 3Anastomotic branches of cricothyroid vessels. 4-Cricotracheal membrane. 5-Anastomotic branches of superior thyroid vessels. 6 Thyroid isthmus. (Reproduced, with permission, from Allas of Topographical and Applied Human Anatomy, W.B. Saunders Company. Vol. 1, 1963.)

tiguous margins of the thyroid and cricoid cartilages. Its upper part, however, is crossed by a small arterial arch formed by the junction of the two cricothyroid arteries; rupture of this artery by the advancing needle may result in a haematoma or troublesome bleeding. Furthermore, if the Tuohy needle misses the midline, it can damage the lateral part of the cricovocal membrane, which is a thinner mernbrane attached to the cricoid, thyroid, and vocal process of the arytenoid cartilages, and laterally forms the vocal Jigament. The thyroid isthmus usually covers the second and third rings of the trachea. It is therefore avoided if the puncture site remains above that level. This higher puncture site will also prevent damage to the anastomotic branch of the two superior thyroid arteries, which nuns along the upper border of the thyroid isthmus (Figure 3). The second advantage of the cricotracheal membrane site is that it allows the tracheal tube to be advanced past the vocal cords before the catheter guide is removed.

Using the catheter to pull as well as guide the tracheal tube down the larynx offers unique advantages: it is safe, surprisingly fast, relatively atraumatic, easy to perform, eliminates most causes of failure, and avoids excessive tension on the guiding catheter. Most important, the tube does not catch on delicate laryngeal structures and does not kink or slip into the oesophagus.

We have now extended the use of this technique to both oral and nasal intubation and in all circumstances of hazardous laryngeal exposures. We employed it in 36 consecutive patients with critical cervical lesions, and apart from minor bleeding at the site of puncture and a few self-limited epistaxes, we have encountered no failures or complications. However, a disturbing problem would arise if, in spite of the gliding knot, the catheter failed to yield or tore inside the tube. We therefore recommend the use of epidural catheters with stylets and the avoidance of forceful pulling. In the event that the catheter is difficult to withdraw, it can safely be secured at the upper end of the tracheal tube and removed at extubation together with the tibe. It is important at this stage to check that the whole of the sut epidural catheter has been removed. As bleeding constitutes a common complication of retrograde guided intubation, it is suggested that the technique be avoided in patients with a pre-existing history of haemorrhagic diathesis.

In conclusion, we believe that the modifications we suggest eliminate most of the technical difficulties of translaryngeal intubation. The technique is safe, well tolerated, and should be used in patients with intubation problems.

\section{Acknowledgements}

The authors are indebted to Dr. Victoria Lees for editorial assistance and to Miss Angie Giannakopoulos for her secretarial help.

\section{References}

1 Akinyemi $O O$. Complications of guided blind endotracheal intubation. Anaesthesia 1979; 34: 590-2.

2 Butler FS, Cirillo AA. Retrograde tracheal intubation. Anesth Analg (Cleve) 1960; 39: 333-8.

3 Findlay $C W$ Jr, Gissen AJ. A guided nasotracheal method for insertion of an endotracheal tube. Anesth Analg 1961; 40: 640-2.

4 Waters $D J$. Guided blind endotracheal intubation: for patients with deformities of the upper airway. Anaesthesia $1963 ; 18$ : $158-62$. 
5 Powell WF, Ozdil T. A translaryngeal guide for tracheal intubation. Anesth Analg 1967; 46: 231-4.

6 Bourke $D$, Levesque $P R$. Modification of retrograde guide for endotracheal intubation. Anesth Analg 1974; 53 :

$1013-4$.

7 Roberts KW. New use for Swan-Ganz introducer wire. Anesth Analg 1981; 60: 67.

8 Borland $L M$, Swan DM, Leff S. Difficult pediatric endotracheal intubation: a new approach to the retrograde technique. Anesthesiology 1981; 55: 577-8.

9 Tobias $R$. Increased success with retrograde guide for endotracheal intubation. Anesth Analg 1983; 62: 366-7.

10 Casthely PA, Fyman PN, Ergin MA, Griepp $R$, Wolf $G L$. Retrograde intubation in patients undergoing open heart surgery. Canad Anaesth Soc J 1985; 32: 661-4.

11 Freund $P R$, Rooke A, Schwid $H$. Retrograde intubation with a modified Eschmann stylet. Anesth Analg 1988; 67: 605-6.
Résumé

La technique de l' intubation trachéale par voie rétrograde a été modifiée afin d' utiliser le catheter, fixé par un noeud coulissant à l'extrémité distale du tube, pour engager celui-ci dans la trachée plutôt que pour le guider. La rapidité et la facilité de la technique réduisent considérablement les causes d'échec et de traumatisme des voies aeriennes. 